\section{A saúde dos trabalhadores da atividade rural no Brasil}

\author{
Rural workers' health in Brazil \\ La salud de los trabajadores dentro de la
actividad rural en Brasil
}

\author{
1 Universidade Federal do \\ Rio de Janeiro, Rio e Janeiro, \\ Brasil. \\ Correspondência \\ J. P. L. Moreira \\ Instituto de Estudos de \\ Saúde Coletiva, Universidade \\ Federal do Rio de Janeiro. \\ Rua Joaquim Rego 29, \\ Rio de Janeiro, $R J$ \\ 21021-590, Brasil. \\ jessica@iesc.ufrj.br
}

\section{Abstract}

Workers' health is a central theme in public health surveys, but the specificity of work activities should be considered. This study aimed to analyze the health of rural workers in Brazil that perform both agricultural and non-agricultural work, based on self-rated health and self-reported diseases. The Brazilian National Household Sample Survey (PNAD 2008) was used, incorporating information from the complex sampling plan. Agricultural workers 18 years or older were selected, stratified according to those with and without non-agricultural work. Logistic regression was performed for self-rated health, and odds ratios were calculated for self-reported diseases. Exclusive agricultural work decreased the odds of reporting good health and increased the odds of reporting back pain, high blood pressure, and arthritis/rheumatism. Exclusive agricultural workers reported more diseases and worse living conditions. Self-rated health was generally better in workers with non-agricultural occupations.

Rural Population; Occupational Health; Morbidity
Jessica Pronestino de Lima Moreira 1

Bruno Luciano Carneiro Alves de Oliveira 1

Camila Drumond Muzi 1

Carlos Leonardo Figueiredo Cunha 1 Alexandre dos Santos Brito 1 Ronir Raggio Luiz 1

\section{Resumo}

A saúde do trabalhador é tema central em pesquisas de saúde pública, entretanto se deve considerar a especificidade das atividades exercidas. Objetiva-se analisar a saúde dos trabalhadores da atividade agrícola no Brasil, que possuem ocupação também agrícola ou não agrícola, por meio da autopercepção de saúde e morbidades referidas. Utilizou-se a Pesquisa Nacional por Amostra de Domicílios (PNAD 2008), incorporando as informações do plano amostral complexo. Selecionaram-se trabalhadores da atividade agrícola com 18 anos ou mais, estratificando em: os que possuíam ocupação agrícola e não agrícola. Foi realizada regressão logística para autopercepção de saúde e calculadas as razões de chances para as morbidades referidas. A ocupação agrícola diminui a chance de referir saúde como Boa e aumenta a chance de referir doença de coluna/costas, hipertensão arterial e artrite/reumatismo. Os trabalhadores com ocupação agrícola apresentam mais morbidades referidas e piores condições de vida. A autopercepção de saúde dos trabalhadores, em geral, foi melhor entre os ocupados não agrícolas.

População Rural; Saúde do Trabalhador; Morbidade 


\section{Introdução}

A saúde dos trabalhadores é condicionada por fatores sociais, econômicos, tecnológicos e organizacionais relacionados ao perfil de produção e consumo, além de fatores de risco de natureza física, química, biológica, mecânica e ergonômica presentes nos processos de trabalho 1 . Os processos produtivos de trabalho implicam diferenciadas inserções dos trabalhadores, com consequente diversificação também dos padrões de morbidade e mortalidade. Esses padrões de saúde e doença são influenciados por fatores decorrentes das condições de vida a que estão submetidos, tanto no meio urbano quanto no rural 2.

Diferentemente do contexto urbano, a população residente no ambiente rural apresenta distintas características em relação à população urbana, tais como: baixa escolaridade e rendimento salarial, difícil acesso dos seus moradores aos serviços sociais, de saúde e comércio, assim como dos profissionais de saúde que atuam nessa área, tendo em vista as distâncias territoriais e a falta de transporte público para deslocamento, tanto dos usuários como da equipe de saúde que a eles assistem ${ }^{1}$. Mas, se por um lado, os trabalhadores agrícolas podem sofrer doenças relacionadas à intensa atividade física no trabalho, à exposição a substâncias tóxicas e à falta de acesso aos serviços de saúde especializados, por outro, têm a vantagem de menor exposição à poluição atmosférica e ao estresse do ambiente urbano. No Brasil, são aproximadamente 30 milhões de trabalhadores submetidos a riscos e agravos das condições de trabalho agrícola, equivalente a cerca de $20 \%$ da população economicamente ativa do país 3 .

A morbidade referida e a autopercepção de saúde são estratégias comumente utilizadas em vários países na mensuração das condições de saúde de diferentes populações. $\mathrm{O}$ indicador autopercepção de saúde descreve, de forma sucinta e eficaz, o estado geral de saúde, considerando aspectos sociais, físicos e mentais 4 do informante. O conceito de autopercepção de saúde expressa o que está por trás da doença, além de ser um poderoso preditor de mortalidade 5. Em estudo realizado em toda a população adulta brasileira, em 2011, verificou-se que $94,4 \%$ da população avaliou seu estado de saúde como bom, muito bom ou regular 6. Em 2001, em estudo realizado entre trabalhadores da indústria na região Sul do Brasil, observou-se que $85,2 \%$ dos indivíduos relatam sua saúde como boa 7 . Em estudo com profissionais de enfermagem na região Centro-Oeste, verificou-se que $77,6 \%$ referiram estado de saúde positivo 8 .
Há uma escassez de estudos que retratem as condições gerais de saúde da população rural no Brasil. No país, em referência aos trabalhadores agrícolas, os estudos existentes são, em sua maioria, direcionados a exposições ou morbidades específicas, como: agrotóxicos 9,10,11,12, ruídos 13, doenças respiratórias 14 e acidentes de trabalho 15. No mundo, o assunto é estudado com maior frequência. Nos EUA, por exemplo, existe uma coorte - Agricultural Health Study - que acompanha um grupo de trabalhadores da agricultura 16 , tendo como resultados inúmeros trabalhos sobre a saúde do trabalhador rural 17,18,19. Tem-se observado no mundo que a exposição a agrotóxicos, a ruídos, maquinaria e outros inerentes ao trabalho agrícola podem contribuir para piores condições de saúde de tais trabalhadores em comparação aos trabalhadores não agrícolas.

A identificação de morbidades que acometem a saúde do trabalhador com ocupação agrícola e das suas condições gerais de saúde, mensurada por meio da autopercepção de saúde, podem revelar os determinantes e condicionantes da saúde expressos no ambiente rural e subsidiar o planejamento de cuidados à saúde de uma parcela importante da população economicamente ativa do Brasil. Ações de promoção, proteção, tratamento e recuperação da saúde devem ser analisadas segundo o contexto social de onde são demandas, pois trabalhadores e moradores da zona rural possuem características distintas na sua forma de viver, trabalhar e se relacionar com o ambiente 20 . Neste sentido, selecionar este ambiente para verificar se existem diferenças entre as ocupações dos trabalhadores faz-se necessário.

O presente estudo pretende verificar se as condições de saúde dos indivíduos com ocupação de trabalho efetivamente agrícola diferenciam-se dos trabalhadores com ocupação não agrícola - seja esta administrativa, científica ou do serviço. Tendo em vista a exposição a fatores específicos da ocupação agrícola, como agrotóxicos e ruídos, optou-se por selecionar apenas trabalhadores de empresas do ramo da atividade agrícola, a fim de proporcionar uma proxy do ambiente rural. Nesse sentido, este artigo tem o objetivo de analisar a autopercepção de saúde e as morbidades autorreferidas entre os trabalhadores da atividade agrícola no Brasil, que possuem ocupação também agrícola ou não agrícola. 


\section{Material e métodos}

\section{População de estudo}

Foram utilizados os dados da Pesquisa Nacional por Amostra de Domicílios (PNAD), de base populacional, realizada pelo Instituto Brasileiro de Geografia e Estatística (IBGE), no ano de 2008, juntamente com o Suplemento Saúde coletado a cada cinco anos, que disponibiliza algumas informações acerca da saúde da população brasileira. Neste ano, foram visitados 150.591 domicílios e participaram do inquérito 391.868 indivíduos. Neste estudo, foram selecionados os trabalhadores com 18 anos ou mais, cuja atividade principal do empreendimento do trabalho era agrícola, na semana de referência para coleta da amostra, compreendida entre os dias 21 a 27 de setembro de 2008, contabilizando 24.018 pessoas.

\section{Variáveis estudadas}

Segundo as notas metodológicas da PNAD 2008 (IBGE), ocupação é definida como o cargo, função, profissão ou ofício exercido pela pessoa. Já atividade do trabalho foi definida como a classificação do empreendimento, obtida por intermédio da finalidade ou do ramo de negócio da organização, empresa ou entidade para a qual a pessoa trabalhava. Para os trabalhadores por conta própria, a classificação foi feita de acordo com a ocupação exercida.

A variável atividade principal do empreendimento do trabalho principal na semana de referência para pessoas de 10 anos ou mais de idade foi definida como variável de seleção, sendo selecionados apenas os trabalhadores cuja atividade do trabalho era agrícola.

Dentre tais trabalhadores de empreendimentos de atividade agrícola, foi feita uma estratificação da variável grupamentos ocupacionais do trabalho principal na semana de referência em dois grupos: ocupação efetivamente agrícola e não agrícola.

Em síntese, a atividade do trabalho refere-se ao ramo da empresa em que a pessoa trabalha, já a ocupação diz respeito ao cargo que a pessoa efetivamente exerce. Há, portanto, um grupo de pessoas que trabalha em empresas do ramo agrícola, que possui ocupação também agrícola e outro grupo da atividade do ramo agrícola, porém, que possui ocupação não agrícola.

Como ocupações agrícolas foram considerados: (1) produtores na exploração agropecuária; (2) trabalhadores na exploração agropecuária; (3) pescadores, caçadores e extrativistas florestais; e (4) trabalhadores da mecanização agropecuária e florestal. Em ocupações não agrícolas foram in- cluídas as seguintes ocupações: (1) dirigentes em geral; (2) profissionais das ciências e das artes; (3) técnicos de nível médio; (4) trabalhadores de serviços administrativos; (5) trabalhadores dos serviços; (6) vendedores e prestadores de serviço do comércio; (7) trabalhadores da produção de bens e serviços e de reparação e manutenção; (8) membros das forças armadas e auxiliares; e (9) ocupações mal definidas.

Consideraram-se as 11 morbidades referidas disponibilizadas pela PNAD, que também foram agrupadas em 4 grupos para serem consideradas as análises: (1) sem morbidade; (2) morbidade tipicamente leve (doença de coluna/costas ou tendinite/tenossinovite); (3) morbidade tipicamente crônica (artrite/reumatismo, diabetes, hipertensão arterial, bronquite/asma ou depressão); e (4) morbidade mais grave (câncer, doença do coração, insuficiência renal crônica ou cirrose). A morbidade Tuberculose não foi levada em conta nas análises por não apresentar frequência entre os trabalhadores com ocupação não agrícola.

O desfecho avaliado foi a autopercepção de saúde que é mensurado por meio de 5 categorias, sendo neste estudo também dicotomizado em boa - abrangendo as categorias muito boa $\mathrm{e}$ boa - e não boa - agrupando as categorias regular, ruim e muito ruim, para utilização na análise múltipla.

Acreditando-se que os trabalhadores com atividade e ocupação agrícolas sejam diferentes dos trabalhadores com atividade agrícola e ocupação não agrícola, as análises para autopercepção de saúde foram controladas pelas variáveis sexo, idade, região geográfica, cor/raça, renda, tabagismo, anos de estudo, posse de plano de saúde e morbidade referida.

\section{Análise estatística}

A amostragem da PNAD é complexa, ou seja, combina métodos de amostragem tradicionais, como a estratificação e a conglomeração, com probabilidades desiguais de seleção em dois ou três estágios - dependendo se os municípios selecionados são ou não autorrepresentativos. Detalhes técnicos sobre o planejamento amostral da PNAD podem ser obtidos em Silva et al. 21

As análises foram realizadas no software estatístico SPSS, versão 17.0 (SPSS Inc., Chicago, Estados Unidos). Considerando o esquema amostral complexo, foram realizados modelos logísticos bivariados e ajustados por todas as variáveis, utilizando como desfecho a autopercepção de saúde, em duas categorias (boa e não boa). Foram incluídas no modelo ajustado todas as variáveis definidas a priori no estudo, mantidas independentemente da significância esta- 
tística. Foram apresentados gráficos de barras para autopercepção de saúde, segundo tipo de ocupação, para a variável em 5 categorias e para a variável dicotomizada. Foi feita a distribuição das características demográficas, socioeconômicas e comportamentais, segundo a ocupação no trabalho. Foram estimadas as prevalências de morbidades referidas entre os trabalhadores e calculadas as razões de chance bruta (OR) para cada morbidade, segundo a ocupação.

Para todas as estimativas geradas, utilizou-se o intervalo de 95\% de confiança (IC95\%).

\section{Resultados}

Dentre os 24.018 trabalhadores de empreendimentos de atividade agrícola, 23.214 trabalhadores possuíam ocupação também agrícola e 804 não agrícola.

A Tabela 1 mostra a distribuição das características demográficas, socioeconômicas e comportamentais dos trabalhadores da atividade agrícola, segundo ocupação exercida. Os trabalhadores com ocupação agrícola, quando comparados aos de não agrícola, são, em maior proporção, do sexo masculino (73,2\%), fumam mais do que os de ocupação não agrícola $(22,4 \% v s$. $15,7 \%$ ) e a maior parte tem a cor/raça não branca $(61,1 \%)$. Quase $30 \%$ dos que possuem ocupação agrícola não têm instrução ou menos de 1 ano de estudo, contra apenas $4,8 \%$ dos trabalhadores com ocupação não agrícola. Ainda entre a população com ocupação agrícola, 15,5\% são idosos, contra apenas $5 \%$ na população com ocupação não agrícola e estão mais concentrados na Região Nordeste do Brasil (47,7\%), seguida pela Região Sudeste $(21,7 \%)$. O contrário é observado entre os trabalhadores com ocupação não agrícola $(47,4 \%$, na Região Sudeste e $22,2 \%$, na Região Nordeste). Maior proporção dos trabalhadores com ocupação agrícola não possuem rendimento ou recebem até 1 salário mínimo $(75,2 \%)$, enquanto entre os não agrícolas a proporção é de $53,0 \%$ (Tabela 1 ).

A Figura la mostra a prevalência de autopercepção de saúde dos trabalhadores, em 5 categorias, segundo a ocupação exercida. Verifica-se que a maioria da população relata ter a saúde boa ou muito boa, em ambas ocupações, entretanto os trabalhadores com ocupação não agrícola, apresentam prevalências ainda maiores. A categoria regular apresenta prevalências elevadas, com diferença grande entre as ocupações, em torno de $30 \%$ entre os trabalhadores agrícolas e de $20 \%$ entre os não agrícolas. Não chega a $1 \%$ a prevalência de trabalhadores com a autopercepção de saúde muito ruim, todavia o dobro da prevalência dos não agrícolas é encontrado entre os trabalhadores agrícolas $(0,6 \%$ vs. $0,3 \%)$ (Figura 1a). A Figura 1b mostra a prevalência de autopercepção de saúde dos trabalhadores dicotomizada, segundo a ocupação exercida. Constata-se que $65,3 \%$ dos trabalhadores agrícolas referem saúde como boa, mas esse percentual é ainda maior entre os não agrícolas (78,3\%) (Figura 1b).

A Tabela 2 apresenta os modelos logísticos para autopercepção de saúde, brutos e ajustados pelas 10 variáveis definidas anteriormente, utilizando a categoria não boa como referência. A ocupação do trabalho agrícola diminui a chance de referir saúde como boa na análise bivariada (OR = 0,52; IC95\%: 0,43-0,64). Na análise multivariada, isso também ocorre, reduzindo somente a magnitude da associação (OR = 0,82; IC95\%: 0,67-1,00).

Com relação às demais variáveis, observa-se que ser do sexo feminino, mais velho e ser fumante ou ex-fumante diminui a chance de referir saúde como boa. Já quanto mais anos de estudo e possuir plano de saúde, aumentam a chance de referir saúde como boa. Em comparação com a Região Norte, residir nas regiões Sul, Sudeste ou Centro-oeste do país aumenta a chance de referir saúde como boa. Encontra-se ainda que, quanto maior a renda do trabalhador, maior a chance de referir saúde como boa. Ter alguma morbidade diminui a chance de referir saúde boa, e essa chance é cada vez menor conforme aumenta a gravidade da doença (Tabela 2).

As prevalências de morbidade referida dos trabalhadores da atividade agrícola no Brasil, segundo a ocupação do trabalho estão apresentadas na Tabela 3. Observa-se que os trabalhadores com ocupação agrícola, em geral, apresentam maiores prevalências de morbidades referidas. As doenças de coluna ou costas $(\mathrm{OR}=$ 1,42; IC95\%: 1,15-1,75), hipertensão arterial (OR = 1,35; IC95\%: 1,07-1,70) e artrite ou reumatismo (OR = 1,79; IC95\%: 1,27-2,54) mostraram maior chance de serem referidas entre esses trabalhadores. As morbidades diabetes $(3,9 \%$ vs. $2,8 \%) \mathrm{e}$ tendinite ou tenossinovite $(2,3 \%$ vs. $1,5 \%)$ foram mais referidas entre os trabalhadores com ocupação não agrícola, contudo o intervalo de confiança inclui o valor 1, sugerindo não associação. Observa-se que o câncer, embora apresente uma prevalência muito pequena, é o dobro entre os trabalhadores com ocupação agrícola em relação aos não agrícolas (Tabela 3).

\section{Discussão}

Os resultados desta análise sugerem diferenças importantes nas características demográficas e 
Distribuição das características demográficas, socioeconômicas e comportamentais dos trabalhadores da atividade agrícola no Brasil, segundo ocupação exercida. Pesquisa Nacional por Amostra de Domicílios (PNAD 2008).

\begin{tabular}{|c|c|c|c|}
\hline \multirow{2}{*}{$\begin{array}{l}\text { Características demográficas, socioeconômicas e } \\
\text { comportamentais }\end{array}$} & \multicolumn{3}{|c|}{ Ocupação agrícola } \\
\hline & $\operatorname{Sim}(\%)$ & Não (\%) & $\begin{array}{c}\text { Total }(\%) \\
(n=24.018)\end{array}$ \\
\hline \multicolumn{4}{|l|}{ Sexo } \\
\hline Masculino & 73,2 & 55,4 & 58,0 \\
\hline Feminino & 26,8 & 44,6 & 42,0 \\
\hline \multicolumn{4}{|l|}{ Faixa etária (anos) } \\
\hline $18-39$ & 46,0 & 59,2 & 57,3 \\
\hline $40-59$ & 38,5 & 35,8 & 36,2 \\
\hline$\geq 60$ & 15,5 & 5,0 & 6,5 \\
\hline \multicolumn{4}{|l|}{ Região } \\
\hline Norte & 7,7 & 7,1 & 7,2 \\
\hline Nordeste & 47,7 & 22,2 & 25,9 \\
\hline Sudeste & 21,7 & 47,4 & 43,6 \\
\hline Sul & 16,7 & 15,6 & 15,8 \\
\hline Centro-oeste & 6,2 & 7,7 & 7,5 \\
\hline \multicolumn{4}{|l|}{ Cor/Raça } \\
\hline Branca & 38,9 & 48,2 & 49,9 \\
\hline Não branca & 61,1 & 51,8 & 50,1 \\
\hline \multicolumn{4}{|l|}{ Faixa de rendimento (salário mínimo) } \\
\hline Sem rendimento até 1 & 75,2 & 53,0 & 74,5 \\
\hline $1-5$ & 20,8 & 41,0 & 21,5 \\
\hline$>5$ & 1,2 & 5,0 & 1,3 \\
\hline Sem declaração & 2,8 & 1,0 & 2,7 \\
\hline \multicolumn{4}{|l|}{ Tabagismo } \\
\hline Não fumante & 46,3 & 56,9 & 55,4 \\
\hline Fumante & 22,4 & 15,7 & 16,6 \\
\hline Ex-fumante & 17,7 & 13,3 & 14,0 \\
\hline Não sabe ou não informou & 13,6 & 14,1 & 14,0 \\
\hline \multicolumn{4}{|l|}{ Anos de estudo } \\
\hline Sem instrução ou menos de 1 & 28,0 & 4,8 & 27,5 \\
\hline $1-7$ & 53,6 & 27,2 & 53,5 \\
\hline$\geq 8$ & 18,3 & 67,8 & 18,9 \\
\hline Não determinado & 0,1 & 0,2 & 0,1 \\
\hline \multicolumn{4}{|l|}{ Posse de plano de saúde } \\
\hline Sim & 5,9 & 34,0 & 29,9 \\
\hline Não & 94,1 & 66,0 & 70,1 \\
\hline
\end{tabular}

comportamentais entre os trabalhadores de atividades agrícolas, tanto os de ocupação agrícola e não agrícola no Brasil em 2008, e um possível perfil de saúde e de doenças associadas à atividade agrícola desenvolvida no país, refletidas na percepção de saúde e nas morbidades referidas. Os trabalhadores que realizavam atividades agrícolas em 2008 eram, em maior proporção, homens, em idade jovem, não brancos, com menor nível de escolaridade, de renda e residentes nas regiões com piores indicadores sociais e de saúde do país. Com relação às morbidades estudadas, percebeu-se que morbidades como doença de coluna ou costas, artrite e reumatismo e hipertensão arterial sistêmica estão associadas à atividade agrícola desenvolvidas no Brasil e que esse padrão de morbidade pode decorrer da intensa carga de esforço físico no trabalho, típicos das atividades laborais musculoesqueléticas, o que repercute nas condições de saúde da população 
Figura 1

Prevalência de autopercepção de saúde dos trabalhadores da atividade agrícola no Brasil ( $n=24.018$ ), segundo a ocupação exercida, em 5 categorias (Figura 1a) e dicotomizada (Figura 1b). Pesquisa Nacional por Amostra de Domicílios (PNAD 2008).

1a) Categorias

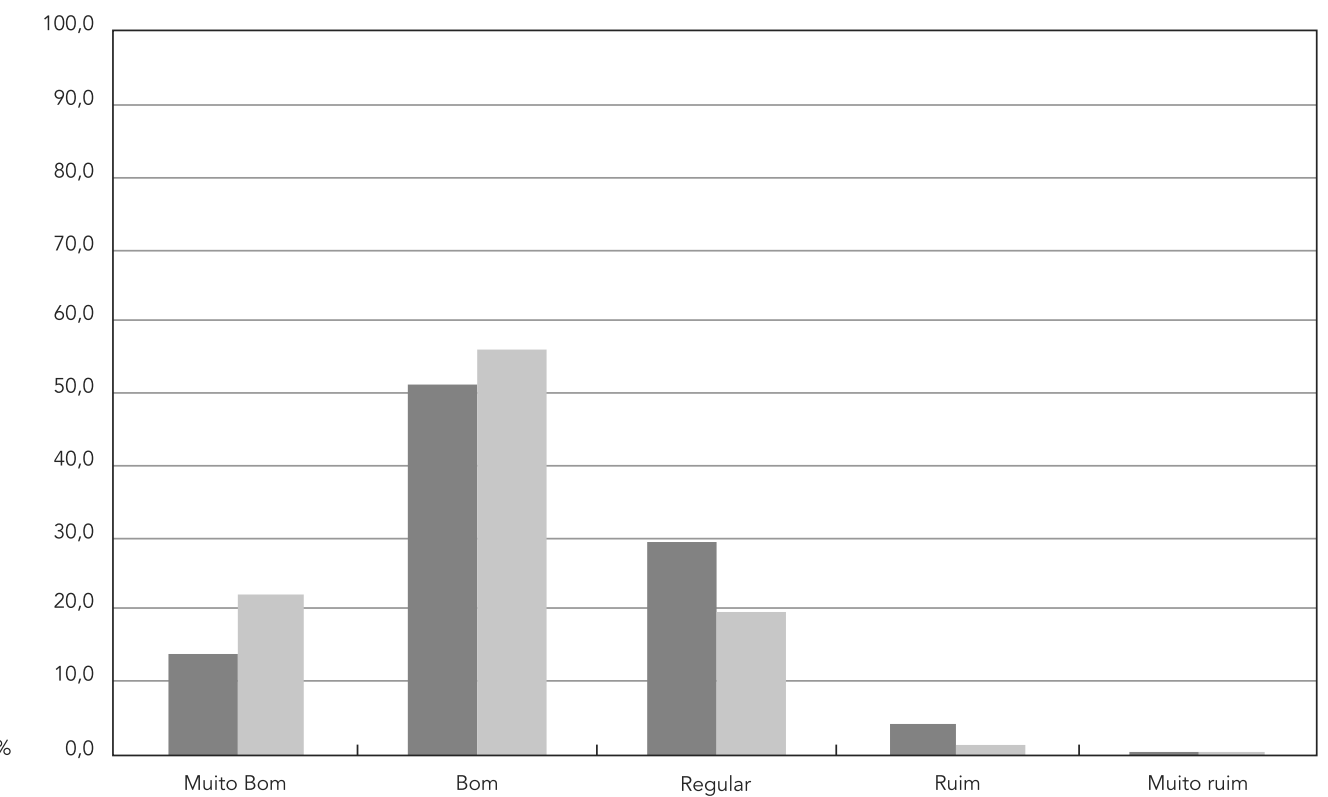

1b) Dicotomizada

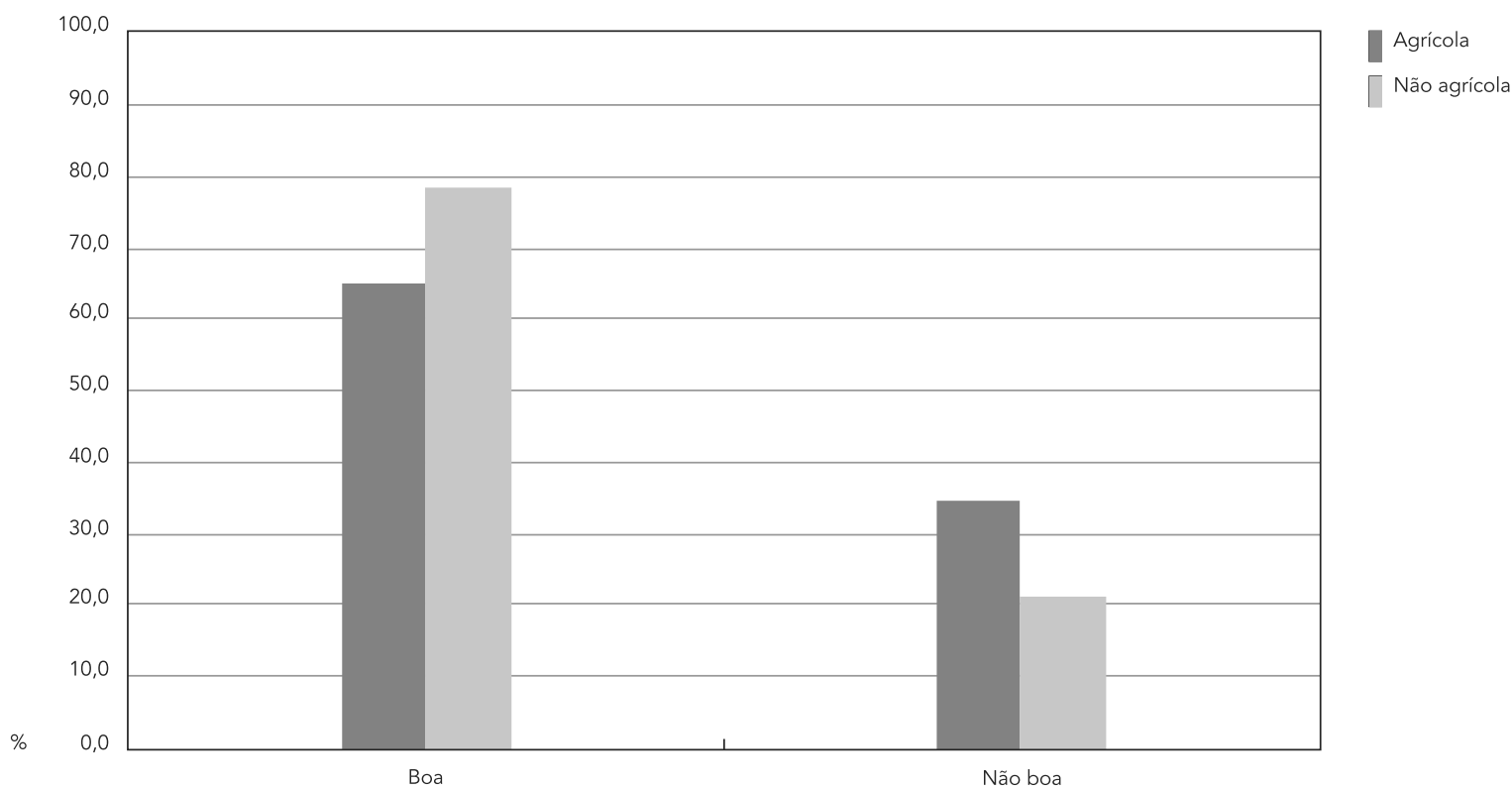


Tabela 2

Modelos logísticos brutos e ajustados para autopercepção de saúde (boa/não boa). Pesquisa Nacional por Amostra de Domicílios (PNAD 2008) $(n=24.018)$.

\begin{tabular}{|c|c|c|c|c|}
\hline \multirow{2}{*}{$\begin{array}{l}\text { Morbidade referida, características } \\
\text { demográficas e comportamentais }\end{array}$} & \multicolumn{4}{|c|}{ Autopercepção de saúde boa * } \\
\hline & OR $_{\text {bruto }}$ & IC $95 \%$ bruto & OR $_{\text {ajustado }}$ & IC95\% ${ }_{\text {ajustado }}$ \\
\hline \multicolumn{5}{|l|}{ Ocupação do trabalho } \\
\hline Agrícola & 0,52 & $0,43-0,64$ & 0,82 & $0,67-1,00$ \\
\hline Não agrícola & 1,00 & & 1,00 & \\
\hline \multicolumn{5}{|l|}{ Sexo } \\
\hline Masculino & 1,00 & & 1,00 & \\
\hline Feminino & 0,60 & $0,56-0,64$ & 0,74 & $0,68-0,81$ \\
\hline \multicolumn{5}{|l|}{ Faixa etária (anos) } \\
\hline $18-39$ & 1,00 & & 1,00 & \\
\hline $40-59$ & 0,35 & $0,32-0,37$ & 0,55 & $0,51-0,60$ \\
\hline$\geq 60$ & 0,24 & $0,22-0,26$ & 0,49 & $0,43-0,55$ \\
\hline \multicolumn{5}{|l|}{ Região geográfica } \\
\hline Norte & 1,00 & & 1,00 & \\
\hline Nordeste & 1,16 & $0,99-1,35$ & 1,20 & $0,99-1,45$ \\
\hline Sudeste & 1,56 & $1,31-1,85$ & 1,73 & $1,42-2,12$ \\
\hline Sul & 1,13 & $0,93-1,36$ & 1,34 & $1,07-1,68$ \\
\hline Centro-oeste & 1,46 & $1,23-1,74$ & 1,52 & $1,25-1,85$ \\
\hline \multicolumn{5}{|l|}{ Cor/Raça } \\
\hline Branca & 1,06 & $0,98-1,14$ & 1,06 & $0,97-1,16$ \\
\hline Não branca & 1,00 & & 1,00 & \\
\hline \multicolumn{5}{|l|}{ Faixa de rendimento (salário mínimo) } \\
\hline Sem rendimento até 1 & 1,00 & & 1,00 & \\
\hline $1-5$ & 1,04 & $0,96-1,13$ & 1,40 & $1,28-1,54$ \\
\hline$>5$ & 1,97 & $1,50-2,60$ & 2,84 & $2,11-3,82$ \\
\hline Sem declaração & 0,92 & $0,76-1,11$ & 1,11 & $0,91-1,36$ \\
\hline \multicolumn{5}{|l|}{ Tabagismo } \\
\hline Não fumante & 1,00 & & 1,00 & \\
\hline Fumante & 0,73 & $0,68-0,79$ & 0,87 & $0,80-0,95$ \\
\hline Ex-fumante & 0,49 & $0,46-0,53$ & 0,79 & $0,72-0,87$ \\
\hline Não sabe ou não informou & 0,76 & $0,71-0,82$ & 0,92 & $0,84-1,00$ \\
\hline \multicolumn{5}{|l|}{ Anos de estudo } \\
\hline Sem instrução ou < 1 & 1,00 & & 1,00 & \\
\hline $1-7$ & 1,47 & $1,37-1,57$ & 1,06 & $0,98-1,16$ \\
\hline$\geq 8$ & 3,04 & $2,76-3,36$ & 1,57 & $1,39-1,77$ \\
\hline Não determinado & 1,23 & $0,61-2,47$ & 0,58 & $0,26-1,29$ \\
\hline \multicolumn{5}{|l|}{ Plano de saúde } \\
\hline Sim & 1,15 & $1,00-1,33$ & 1,20 & $1,02-1,40$ \\
\hline Não & 1,00 & & 1,00 & \\
\hline \multicolumn{5}{|l|}{ Morbidade referida } \\
\hline Sem morbidade & 1,00 & & 1,00 & \\
\hline Morbidade tipicamente leve & 0,24 & $0,22-0,27$ & 0,28 & $0,25-0,30$ \\
\hline Morbidade tipicamente crônica & 0,14 & $0,13-0,16$ & 0,18 & $0,16-0,19$ \\
\hline Morbidade mais grave & 0,08 & $0,07-0,09$ & 0,09 & $0,08-0,10$ \\
\hline
\end{tabular}

IC95\%: intervalo de 95\% de confiança; OR: odds ratio.

* Autopercepção de saúde boa vs. não boa 


\begin{tabular}{|c|c|c|c|c|c|}
\hline \multirow[t]{2}{*}{ Morbidade referida } & \multicolumn{2}{|c|}{ Ocupação agrícola } & \multirow{2}{*}{$\begin{array}{c}\text { Total }(\%) \\
(n=24.018)\end{array}$} & \multirow[t]{2}{*}{ OR } & \multirow[t]{2}{*}{ IC95\% } \\
\hline & $\operatorname{Sim}(\%)$ & Não (\%) & & & \\
\hline Doença de coluna ou costas & 21,1 & 15,8 & 20,9 & 1,42 & $1,15-1,75$ \\
\hline Artrite ou reumatismo & 7,7 & 4,5 & 7,6 & 1,79 & $1,27-2,54$ \\
\hline Câncer & 0,6 & 0,3 & 0,5 & 2,15 & $0,65-7,13$ \\
\hline Diabetes & 2,8 & 3,9 & 2,9 & 0,75 & $0,50-1,03$ \\
\hline Bronquite ou asma & 2,9 & 2,4 & 2,9 & 1,23 & $0,79-1,91$ \\
\hline Hipertensão arterial & 17,3 & 13,4 & 17,2 & 1,35 & $1,07-1,70$ \\
\hline Doença do coração & 4,0 & 2,7 & 4,0 & 1,51 & $0,97-2,35$ \\
\hline Insuficiência renal crônica & 2,0 & 1,9 & 2,0 & 1,08 & $0,63-1,87$ \\
\hline Depressão & 3,7 & 2,5 & 3,6 & 1,47 & $0,90-2,39$ \\
\hline Tendinite ou tenossinovite & 1,5 & 2,3 & 1,5 & 0,63 & $0,37-1,08$ \\
\hline Cirrose & 0,3 & 0,2 & 0,3 & 1,08 & $0,24-4,73$ \\
\hline
\end{tabular}

IC95\%: intervalo de 95\% de confiança; OR: odds ratio.

estudada. Entre os trabalhadores avaliados, diferenças importantes foram encontradas nas estimativas de autopercepção de saúde, nas quais os trabalhadores não agrícolas referiram melhores estados de saúde, do que os trabalhadores agrícolas, refletindo a possível influência dos fatores de risco, intrínsecos a esse tipo de trabalho (exposições a agrotóxicos, ruídos entre outros).

Em todo o mundo, tem crescido o interesse na saúde de populações vivendo em áreas rurais e remotas e na organização de serviços de saúde para atender a essas populações 22. Estudos realizados nos Estados Unidos, Austrália, Canadá e Escócia têm constatado que moradores de área rural apresentam pior estado de saúde autorreferido do que os moradores de área urbana e que a área rural parece ser o principal determinante do padrão de saúde, acesso e organização de serviços de saúde 22 .

Semelhantes a estudos desenvolvidos em outros países, encontrou-se que características demográficas e socioeconômicas da população que realiza atividade agrícola estão associadas com piores condições de saúde em áreas rurais22. Na análise da variável sexo, foi observado que a maioria dos trabalhadores com ocupação agrícola do Brasil pertencia ao sexo masculino, assim como observado em outros estudos 20,23, ainda que superando quase em $20 \%$ esses estudos. Analisando-se a variável anos de estudo, verifica-se uma predominância entre 1 a 7 anos, assim como encontrado em estudo realizado na região Centro-oeste 24 . Em estudo realizado no Nordeste, observou-se mais de um terço de trabalhadores sem instrução e com menos de 1 ano de estudo 25 , enquanto no Sul do país, foi constatado um percentual muito baixo de analfabetismo, em torno de $5 \% 20,23$.

É preciso observar que as alterações nas relações de trabalho no campo não ocorrem homogeneamente em todo o país. Nas regiões mais desenvolvidas, como regiões Sul e Sudeste, elas ocorrem com maior intensidade e em menor tempo; por outro lado, nas demais regiões brasileiras, pode-se identificar a intensificação tecnológica apenas em algumas propriedades 26 . Nota-se que experiência e bons antecedentes, ainda são os critérios mais apontados para seleção de trabalhadores rurais que atuam diretamente nas atividades agropecuárias, em detrimento da alfabetização e procedência, conforme aponta estudo realizado no Mato Grosso do Sul 24.

Na Finlândia, em um estudo comparativo entre os trabalhadores da agricultura e administrativos, foram encontradas maiores prevalências de doenças crônicas entre os agricultores acima de 40 anos de idade 27. Em estudo sobre os agricultores nesse mesmo país, observou-se uma prevalência de doenças crônicas em torno de $40 \%$, hierarquicamente em doenças musculoesqueléticas, doença cardiovascular, respiratórias e de pele 28 . Na Coreia do Sul, os agricultores apresentaram maior prevalência de artrite, hipertensão e problemas de coluna e menor prevalência de diabetes mellitus, câncer e catarata/glaucoma, comparados com outras populações ${ }^{29}$. Maiores prevalências de artrite, hipertensão e problemas de coluna foram observados entre os 
trabalhadores com ocupação agrícola neste estudo, concordando com o estudo realizado na Coreia, entretanto doenças como câncer e diabetes também foram mais prevalentes entre os trabalhadores agrícolas.

Em contrapartida, em estudo prospectivo na Suíça, assumindo três grupos de trabalhadores: agricultores, outros trabalhadores rurais e trabalhadores urbanos, verificou-se que, em geral, os trabalhadores agrícolas, tanto agricultores como outros trabalhadores rurais apresentam morbidade por doenças crônicas, em geral, menor do que os trabalhadores urbanos. Quando se compararam apenas os agricultores com os outros trabalhadores rurais, não foi verificada diferença de morbidade entre eles, considerando todas as causas. Para causas específicas, como doenças psiquiátricas e cardiovasculares, os agricultores apresentaram menor morbidade 30 . No entanto, no presente estudo, em que os trabalhadores rurais e os agricultores foram agrupados em uma única categoria, maior morbidade foi encontrada neste grupo.

Analisando todos trabalhadores em ocupação agrícola e não agrícola, independentemente da atividade do trabalho, e também com os dados da PNAD 2008, Maia \& Rodrigues 31 demonstra que, em geral, os ocupados da atividade agrícola possuíam maior probabilidade de declarar boa saúde. Tal resultado difere deste estudo, que encontrou que os trabalhadores com ocupação agrícola têm menor chance de declarar a saúde como boa, salientando que no presente trabalho foram levados em conta apenas os trabalhadores que possuem atividade agrícola.

Um dos pontos positivos é que, diferentemente de outras pesquisas que usam agregados rural vs. urbano, este estudo desagregou a população do ambiente rural e buscou verificar as condições de saúde de pessoas que trabalham em atividades agrícolas, comparando os que possuem uma ocupação agrícola com os não agrícola. Além disso, para permitir maior comparação entre os grupos, sob a hipótese de que, dessa forma, possibilitaria controlar também pelas condições do ambiente, foram selecionados apenas os trabalhadores da atividade agrícola.

Porém, selecionar os trabalhadores da atividade agrícola não garante que tais trabalhadores compartilhem do mesmo ambiente, tendo em vista que os trabalhadores de empreendimentos de atividade agrícola, mas com ocupação não agrícola, não necessariamente encontram-se no ambiente rural, o que pode ser considerado como uma limitação do estudo.

O presente estudo permitiu conhecer um pouco sobre as condições de saúde dos trabalhadores agrícolas e como eles percebem sua própria saúde, de modo que possa ser útil para potenciais políticas públicas e para fins de comparação em avaliações dessas políticas no futuro, motivado por estudos específicos.

\section{Resumen}

La salud del trabajador es el tema central en investigaciones en salud pública, sin embargo, se debe considerar la especificidad de las actividades ejercidas. Se trata de analizar la salud de los trabajadores dentro del sector agrícola en Brasil, que poseen una ocupación también agrícola o no agrícola, por medio de la autopercepción de salud y morbilidades referidas. Se utilizó la Encuesta Nacional por Muestra de Domicilios (PNAD 2008), incorporando la información del diseño por muestra del complejo. Se seleccionaron trabajadores del sector agrícola con 18 años o más, estratificando entre los que poseían una ocupación agrícola y no agrícola. Se realizó una regresión logística para autopercepción de salud y se calcularon las razones de posibilidades para las morbilidades referidas. La ocupación agrícola disminuye la posibilidad de informar sobre la salud positivamente y aumenta la posibilidad de informar sobre enfermedades de la columnalespalda, hipertensión arterial y artritis/reumatismo. Los trabajadores con ocupación agrícola presentan más morbilidades referidas y peores condiciones de vida. La autopercepción de salud de los trabajadores, en general, fue mejor entre quienes cuentan con una ocupación no agrícola.

Población Rural; Salud Laboral; Morbilidad 


\section{Colaboradores}

J. P. L. Moreira, B. L. C. A. Oliveira, C. D. Muzi, C. L. F. Cunha, A. S. Brito e R. R. Luiz participaram de todas as etapas de elaboração deste artigo.

\section{Referências}

1. Dias EC. Condições de vida, trabalho, saúde e doença dos trabalhadores rurais no Brasil. In: Pinheiro TMM, organizador. Saúde do trabalhador rural-RENAST. Brasília: Ministério da Saúde; 2006. p. 1-27.

2. Minayo-Gomez C, Thedim-Costa SMF. A construção do campo da saúde do trabalhador: percurso e dilemas. Cad Saúde Pública 1997; 13 Suppl 2:S21-32.

3. Instituto Brasileiro de Geografia e Estatística. Anuário estatístico do Brasil. Rio de Janeiro: Instituto Brasileiro de Geografia e Estatística; 2012.

4. Lang T, Delpierre C. 'How are you?': what do you mean? Eur J Public Health 2009; 19:353.

5. Idler EL, Benyamini Y. Self-rated health and mortality: a review of twenty-seven community studies. J Health Soc Behav 1997; 38:21-37.

6. Moraes JR, Lima Moreira JP, Luiz RR. Association between self-reported state of health among adults and the location of the home: ordinal logistic regression analysis using PNAD 2008. Ciênc Saúde Coletiva 2011; 16:3769-80.

7. Barros MVG, Nahas MV. Health risk behaviors, health status self-assessment and stress perception among industrial workers. Rev Saúde Pública 2001; 35:554-63.

8. Theme Filha MM, Costa MAS, Guilam, MCR. Occupational stress and self-rated health among nurses. Rev Latino-Am Enfermagem 2013; 21:475-83.

9. Faria NMX, Facchini LA, Fassa AG, Tomasi E. Trabalho rural e intoxicações por agrotóxicos. Cad Saúde Pública 2004; 20:1298-308.

\section{Agradecimentos}

Este projeto foi parcialmente financiado pela FAPERJ (processos no E-26/100.682/2007 e E-26/101.506/2010).
10. Silva JM, Novato-Silva E, Faria HP, Pinheiro TMM Agrotóxico e trabalho: uma combinação perigosa para a saúde do trabalhador rural. Ciênc Saúde Coletiva 2005; 10:13.

11. Domingues MR, Bernardi MR, Ono EYS, Ono MA. Agrotóxicos: Risco à Saúde do Trabalhador Rural. Semina: Ciências Biológicas e da Saúde. 2004;25:10.

12. Soares W, Almeida RMVR, Moro S. Trabalho rural e fatores de risco associados ao regime de uso de agrotóxicos em Minas Gerais, Brasil. Cad Saúde Pública 2003; 19:1117-27.

13. Gosling M, Araújo GCD. Saúde física do trabalhador rural submetido a ruídos e à carga térmica: um estudo em operadores de tratores. Mundo Saúde 2008; 32:275-86.

14. Viegas CAA. Agravos respiratórios decorrentes da atividade agrícola. J Pneumologia 2000; 26:8.

15. Fehlberg MF, Santos I, Tomasi E. Prevalência e fatores associados a acidentes de trabalho em zona rural. Rev Saúde Pública 2001; 35:269-75.

16. National Institute of Environmental Health Sciences. Agricultural Health Study (AHS). http:// aghealth.nih.gov/ (acessado em 23/Mai/2014).

17. Blair A, Tarone R, Sandler D, Lynch C, Rowland A, Wintersteen W, et al. Reliability of reporting on life-style and agricultural factors by a sample of participants in the Agricultural Health Study from Iowa. Epidemiology 2002; 13:95-9. 
18. De Roos AJ, Blair A, Rusiecki JA, Hoppin JA, Svec $\mathrm{M}$, Dosemeci $\mathrm{M}$, et al. cancer incidence among glyphosate-exposed pesticide applicators in the Agricultural Health Study. Environ Health Perspect 2005; 113:49-54

19. Alavanja MC, Samanic C, Dosemeci M, Lubin J, Tarone R, Lynch CF, et al. Use of agricultural pesticides and prostate cancer risk in the Agricultural Health Study Cohort. Am J Epidemiol 2003; 157:800-14.

20. Severo LO, Vaz MRC, Costa VZ, Simon D, Rocha LP, Borges AM. Enfermagem e o contexto rural: relações com a saúde, ambiente e trabalho. Revista de Enfermagem UFPE On Line 2012; 6:2950-8.

21. Silva PLN, Pessoa DGC, Lila MF. Análise estatística de dados da PNAD: incorporando a estrutura do plano amostral. Ciênc Saúde Coletiva 2002; 7 : 659-70.

22. Teckle P, Hannaford P, Sutton M. Is the health of people living in rural areas different from those in cities? Evidence from routine data linked with the Scottish Health Survey. BMC Health Services Research 2012; 12:43.

23. Faria NMX, Facchini LA, Fassa AG, Tomasi E. Processo de produção rural e saúde na serra gaúcha: um estudo descritivo. Cad Saúde Pública 2000; 16:115-28.

24. Arruda EE, Figueiredo Neto LF, Fachini JC, Fachini MÂ, Mendonça PSM. Perfil do trabalhador rural da pecuária e da agricultura na Região de Sidrolândia - MS. In: Anais do XLIII Congresso da Sociedade Brasileira de Economia e Sociologia Rural Ribeirão Preto. http://www.sober.org.br/palestra/2/924.pdf (acessado em 14/Jun/2014).
25. Alves CLB, Paulo EM. Mercado de trabalho rural cearense: evolução recente a partir dos dados da PNAD. Revista da ABET 2012; XI:47-61.

26. Elias D. A globalização e modernização agrícola. Revista Paranaense de Geografia 1996; 1:14-29.

27. Notkola V, Husman KR, Susitaival P, Taattola K. Morbidity and risk factors of Finnish farmers. Scand J Work Environ Health 1992; 18 Suppl 2:51-4.

28. Perkiö-Mäkelä MM. Finnish farmers' self-reported morbidity, work ability, and functional capacity. Ann Agric Environ Med 2000; 7:11-6.

29. Lee WJ, Cha ES, Moon EK. Disease prevalence and mortality among agricultural workers in Korea J Korean. Med Sci 2010; 25:7.

30. Thelin N, Holmberg S, Nettelbladt P, Thelin A. Mortality and Morbidity among Farmers, Nonfarming Rural Men, and Urban Referents. A Prospective Population-Based Study. Int J Occup Environ Health 2009; 15:8.

31. Maia AG, Rodrigues CG. Saúde e mercado de trabalho no Brasil: diferenciais entre ocupados agrí colas e não agrícolas. Revista de Economia e Sociologia Rural 2010; 48:737-65.

Recebido em 09/Jul/2014

Versão final reapresentada em 03/Fev/2015

Aprovado em 02/Mar/2015 